\title{
THE RESPONSE OF NANOSTRUCTURED SURFACES IN THE NEAR FIELD
}

\section{ARISING FROM: P. LALANNE \& J. P. HUGONIN, NATURE PHYS. 2, 551-558 (2006)}

To the Editors - The authors of ref. 1 state that their numerical calculation of the transmission intensity dependence of very simple subwavelength planar structures in a silver film agrees well with an earlier-developed model ${ }^{2}$, but both model and numerical simulation show significant disagreement with the experimental results of ref. 3. The authors ${ }^{1}$ speculate that the silver surfaces of the subwavelength structures used in ref. 3 were contaminated by an $11 \mathrm{~nm}$ overlayer of silver sulphide, because such a layer would bring the reported experimental results and their calculations into better agreement.

We have analysed the physical-chemical surface properties of the single-slit, single-groove subwavelength-structured silver films used in the experiments with high-resolution transmission electron microscopy (HRTEM), and we have calculated fully vectorial numerical solutions to Maxwell's equations for the relevant structures using the finite-difference-time-domain (FDTD) technique ${ }^{4}$. The HRTEM analysis shows that the silver films are free of detectable contaminants with a detection limit orders of magnitude below the $11 \mathrm{~nm}$ layer suggested by ref. 1. Furthermore, the FDTD calculations are in excellent agreement with experiment (Fig. 1a), showing a rapid fringe amplitude decrease in the near-zone (slit-groove distance out to 3-4 wavelengths). Extended FDTD calculations to slit-groove distances beyond the near-zone (Fig. 1b) show that the surface wave evolves to the expected bound surface plasmon polariton (SPP). The key finding is that a surface wave in the near-zone consists of a distribution of transient surface modes adjacent and in addition to the bound SPP. Beyond the near-zone the results confirm that the transients dissipate with only the bound SPP mode surviving. This result has important implications for the interpretation of light transmission through arrays of subwavelength structures with subwavelength pitch. The common assumption that only the SPP mode is populated over the entire surface is not justified in the near-zone.

\section{REFERENCES}

1. Lalanne, P. \& Hugonin, J. P. Nature Phys. 2, 551-558 (2006).

2. Lalanne, P., Hugonin, J. P. \& Rodier, J. C. Phys. Rev. Lett. 95, 263902 (2005).

3. Gay, G. et al. Nature Phys. 2, 262-267 (2006).

4. Gay, G. et al. Preprint at <http://arxiv.org/physics/0608116> (2006)

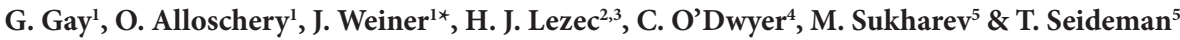

${ }^{1}$ IRSAMC/LCAR Université Paul Sabatier, 118 route de Narbonne, 31062 Toulouse, France.

${ }^{2}$ Applied Physics, Caltech, Pasadena, California 91125, USA.

${ }^{3}$ CNRS, 75794 Paris, France.

${ }^{4}$ Tyndall National Institute, University College Cork, Cork, Ireland.

${ }^{5}$ Department of Chemistry, Northwestern University, Evanston, Illinois 60208, USA.

*e-mail: jweiner@irsamc.ups-tlse.fr

Authors' reponse - Because Maxwell's equations for linear dielectric materials are exact, computation plays a crucial role in the analysis of light scattering by subwavelength structures. In ref. 1, two of the present authors use a fully vectorial method to analyse the normalized coupled power $|S|^{2} /\left|S_{0}\right|^{2}$ into the fundamental propagating slit mode of a slit-groove geometry in a silver film previously studied in ref. 2, see Fig. 1a. In agreement with the experimental results ${ }^{2},|S|^{2} /\left|S_{0}\right|^{2}$ exhibits an oscillatory behaviour as a function of the slit-groove distance $d$ (Fig. 1b). However, the theoretical oscillation frequency, which perfectly matches the propagation constant $k_{\mathrm{SP}}=k_{0}\left(\varepsilon_{\mathrm{Ag}} /\left(\varepsilon_{\mathrm{Ag}}+1\right)\right)^{1 / 2}$ of the $\mathrm{Ag}$-air surface plasmon polariton (SPP), significantly differs from the experimental one. Thus, an SPP-mediated interaction mechanism is promoted in ref 1 , leading to an interpretation very different from that in ref. 2. 